Manuscript received April 8, 2019; accepted for publication January 21, 2020; published online February 14, 2020.

1 Division 8.5 Micro-NDE, Federal Institute for Materials Research and Testing, Unter den Eichen 87, 12205 Berlin, Germany

${ }^{2}$ Division 8.5 Micro-NDE, Federal Institute for Materials Research and Testing, Unter den Eichen 87, 12205 Berlin, Germany (Corresponding author), e-mail: bernd.mueller@bam.de, (1) https://orcid.org/0000-00032234-1538

3 Institute of Materials Research, German Aerospace Center, Linder Hoehe, 51147 Cologne, Germany

${ }^{4}$ Institute for Physics and Astronomy, University of Potsdam, Karl-Liebknecht-Straße 24/25, 14476 Potsdam, Germany, (1) https://orcid.org/0000-00019632-3960
René Laquai, ${ }^{1}$ Bernd Randolf Müller, ${ }^{2}$ Galina Kasperovich, ${ }^{3}$ Guillermo Requena, ${ }^{3}$ Jan Haubrich, ${ }^{3}$ and Giovanni Bruno,

\section{Classification of Defect Types in SLM Ti-6AI-V4 by X-ray Refraction Topography}

\section{Reference}

R. Laquai, B. R. Müller, G. Kasperovich, G. Requena, J. Haubrich, and G. Bruno, "Classification of Defect Types in SLM Ti-6AI-V4 by X-ray Refraction Topography," Materials Performance and Characterization 9, no. 1 (2020): 82-93. https://doi.org/10.1520/MPC20190080

\section{ABSTRACT}

Porosity in additively manufactured materials, such as laser powder bed fusion Ti-Al6-V4, can play an important role in their mechanical performance. Not only the total porosity but also the shape/morphology of the individual pores need to be considered. Therefore, it is necessary to determine the distributions of different defect types (especially fusing defects and keyhole pores) and their dependence on process parameters. We show that synchrotron $X$-ray refraction radiography allows analysis of large samples (up to several millimeters) without compromising the detectability of submicrometer defects. Correspondingly, a classification tool is introduced that is able to quantitatively distinguish defects such as keyhole pores and binding defects with a confidence level of $94 \%$, even when the shape cannot be discerned because of limited spatial resolution.

\section{Keywords}

additive manufacturing, selective laser melting, X-ray refraction, microscopy, porosity, X-ray computed tomography

\section{Introduction}

Laser powder bed fusion methods, like selective laser melting (SLM), have been widely studied during recent years as additive manufacturing (AM) technology allowing the tool-free production of complex configuration components directly from the computeraided design models. ${ }^{1,2}$ However, the rapid solidification during the process and nonoptimized laser process parameters can cause the formation of undesirable defects such as voids during layer-by-layer material building. These voids play an important role for the damage tolerance behavior, especially for cyclically loaded AM components, as shown, for instance in a recent study by Leuders et al. $^{3}$ 
The defect formation has been extensively investigated for Ti-6Al-4V alloy (see among others Vilaro, Colin, and Bartout, ${ }^{4}$ Gong et al., ${ }^{5}$ and Günther et al. ${ }^{6}$ ). The processing-induced porosity can be detrimental for the mechanical performance of the alloy under fatigue conditions, as was experimentally confirmed ${ }^{3,5,7-10}$ and predicted in numerical simulations. ${ }^{11}$

Not only is the total porosity critical for final material properties but also the individual types (shapes) of defects are of great importance in predicting durability and fatigue resistance of Ti- $6 \mathrm{Al}-4 \mathrm{~V}$ parts. ${ }^{12,13}$ The role of porosity and pore shape on the mechanical properties of materials is a classic problem that has been well studied. ${ }^{14,15}$ In the case of additively manufactured metals (with particular focus on Ti-6Al-4V), it has been noted by various researchers, such as Xu et al., ${ }^{16}$ Chastand et al., ${ }^{17}$ and Kasperovich and Hausmann, ${ }^{18}$ that the type of defects is critical to the mechanical behavior of these materials, especially in the case of their cyclic loading.

The generation of defects as a function of the process energy density has been studied in depth and detailed in our previous work. ${ }^{19}$ The pore morphology was characterized in detail by 2-D optical microscopy and 3-D synchrotron computed tomography. ${ }^{19}$ Pore size and orientation, with respect to the build direction, was described in terms of aspect ratio, Feret parameter, and curvature distribution, which allowed the evaluation of the most critical defects. A low (insufficient) energy density increases porosity because of incomplete melting of the powder particles. This, combined with the balling effect and hillocks as well as a lack of fusion, leads to the formation of elongated crack-like pores with sharp and mostly concave boundaries. On the contrary, because of gas bubble formation, a high (excessive) energy density creates spherical vaporization pores, which have the form of a keyhole. They have predominantly convex boundaries and may exhibit sharp rims at their surfaces as a result of the highly dynamic solidification of the SLM process. ${ }^{5,8}$

Optical and electron microscopy yield a great deal of information and provide a necessary data base before undertaking more advanced studies. However, they have inherent limitations: they yield only 2-D views, they are time consuming (including sample preparation), they are destructive, and their field of view is limited. Laboratory and synchrotron X-ray computed tomography (SXCT) in contrast yield 3-D data and are mostly nondestructive but are generally not suited to achieve sufficient resolution for larger components. However, as for microscopy, while synchrotron radiation computed tomography can possess a high spatial resolution, this can only be obtained at the price of a limited field of view (and sometimes a time-consuming sample preparation) and very small sample size. ${ }^{19}$ Analogously, laboratory computed tomography does not allow for observing pores with sizes below its spatial resolution (typically $5-10 \mu \mathrm{m}$ ).

The necessity of alternative and complementary methods for the determination of pore characteristics that would be faster and less labor intensive as well as allow fields of view of "macroscopic size" (say, $1 \mathrm{~cm})$ is apparent.

X-ray refraction techniques ${ }^{20-22}$ are radiographic and tomographic techniques that combine a large field of view (several millimeters) with an exceptional detectability of small defects (whose size was estimated to lie below $1 \mathrm{~nm}$ ). ${ }^{23}$ While their spatial resolution is limited by the beam size in the laboratory (typically 50 by $1,000 \mu \mathrm{m}$ ), and by the camera pixel size at a synchrotron (typically $1-5 \mu \mathrm{m}$ ), they are able to detect nanometric objects. ${ }^{24}$ Moreover, by means of X-ray refraction techniques, it is possible to detect the orientation dependence of the features investigated (in our case, pores, but in general, all "defects," such as grain boundaries, cracks, inclusions, etc.). ${ }^{25}$

$\mathrm{X}$-ray refraction techniques have already been successfully used to distinguish pore shapes in SLM Ti-6Al-4V. ${ }^{26}$ In this study, we will show how the combination of 2-D X-ray refraction and 3-D computed tomography allows for a statistical classification of defects in additively manufactured materials, in the example of Ti-6Al-4V.

\section{Materials and Methods}

\section{SPECIMENS AND MANUFACTURING PARAMETERS}

Plasma atomized Ti-Al6-V4 extra low interstitials powder was received from Advanced Powders \& Coatings (Canada). Cuboids of 10 by 10 by $10 \mathrm{~mm}^{3}$ were produced in an argon atmosphere by an SLM-280 (SLM 
Solutions) machine equipped with a $400 \mathrm{~W}$ laser $(\lambda=1,070 \mathrm{~nm})$ at a building platform temperature of $200^{\circ} \mathrm{C}$. The cuboids were produced without contour parameters. The used SLM volume processing parameter sets, labeled A1 to A10 in this study, consist of different scan velocities at constant values: laser power $=175 \mathrm{~W}$, hatch distance $=100 \mu \mathrm{m}$, layer thickness $=30 \mu \mathrm{m}$, and laser focus $=0 \mathrm{~mm}$. The scan velocities were varied in steps of $100 \mathrm{~mm} \cdot \mathrm{s}^{-1}$ between $200 \mathrm{~mm} \cdot \mathrm{s}^{-1}$ (parameter set A1) to $1,100 \mathrm{~mm} \cdot \mathrm{s}^{-1}$ (parameter set A10). This resulted in volume energy densities ${ }^{19}$ between $292 \mathrm{~J} \cdot \mathrm{mm}^{-3}$ (A1) and $53 \mathrm{~J} \cdot \mathrm{mm}^{-3}$ (A10), which in turn influenced the predominant type of voids/defects. The reader is referred to Kasperovich et al. ${ }^{19}$ and Laquai et al. ${ }^{26}$ for further details on sample manufacturing, materials, and preparation.

At the two limits of the optimum energy density range (between 53 and $195 \mathrm{~J} \cdot \mathrm{mm}^{-3}$ ), different defect types dominate. As already reported elsewhere, ${ }^{5,12,19}$ fusing defects and balling are observed below the lower limit, whereas at higher energy density, vaporization (keyhole) pores form the majority of defects (fig. 1). The balling and fusing defects can have lengths $>100 \mu \mathrm{m}$ and appear crack like. The keyhole pores show diameters of up to about $50 \mu \mathrm{m}$.

The porosity of all cuboids was first measured by means of the Archimedes' method. Successively, samples were prepared from each cuboid for investigation by SXCT and optical microscopy (see also Kasperovich et al. ${ }^{19}$ and Laquai et al. $^{26}$ ).

\section{SYNCHROTRON X-RAY REFRACTION RADIOGRAPHY}

From five cuboids, 0.35 -mm-thick plates cut parallel to the build direction were prepared for investigation by synchrotron X-ray refraction radiography (SXRR). These samples are listed in Table 1 with their respective SLM parameters and the resulting energy density.

SXRR measurements were performed at BAMline at Helmholtz-Zentrum Berlin, Germany. ${ }^{27,28}$ SXRR uses the rocking curve of an analyzer crystal, placed between sample and detector, to gain information about the inner surfaces of the sample. From the maximum and integral intensities $\left(I_{\max }\right.$ and $\left.I_{\text {int }}\right)$ of the rocking curve, the refraction value $C$ is calculated according to equation (1):

$$
C \cdot d=1-\frac{I_{\max } \cdot I_{\text {int }, 0}}{I_{\max , 0} \cdot I_{\text {int }}}
$$

where $d$ is the sample thickness, and the index 0 refers to measurement without the sample. The refraction value $C$ is proportional to the internal specific surface, i.e., the surface per unit volume. To eliminate the influence

FIG. 1 Examples of defect types predominant in different regions of energy density and their appearance in different image modalities: scanning electron microscopy (SEM), SXCT, and SXRR.

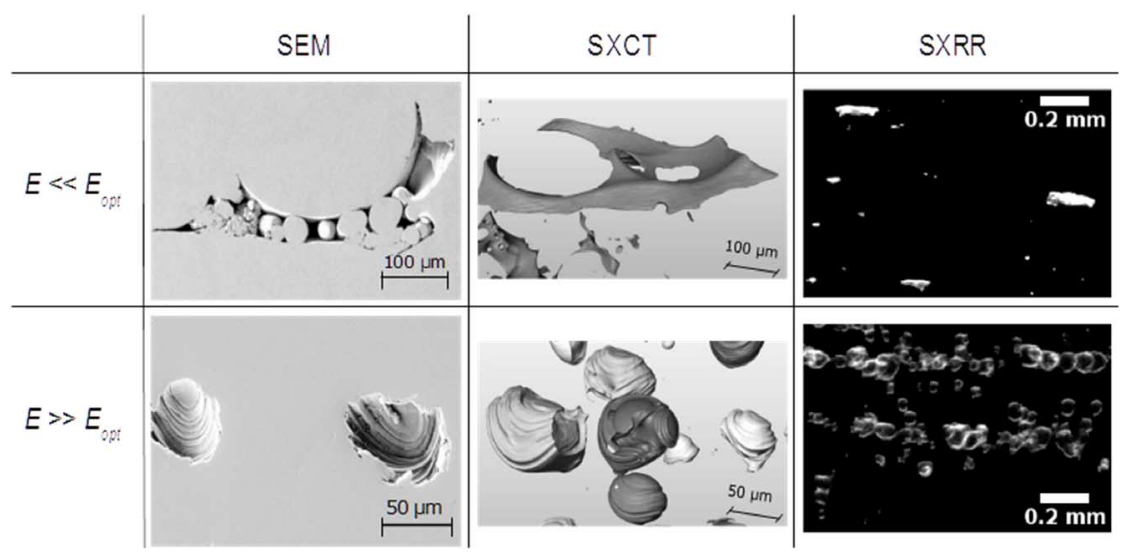




\section{TABLE 1}

Samples investigated by SXRR with respective scan velocity and resulting energy density (all other parameters remained constant)

\begin{tabular}{lcc}
\hline Label & Scan Velocity, $\mathrm{mm} \cdot \mathrm{s}^{-1}$ & Energy Density, J.mm $^{-3}$ \\
\hline A1 & 200 & 292 \\
A2 & 300 & 195 \\
A4 & 500 & 117 \\
A7 & 800 & 73 \\
A10 & 1,100 & 53 \\
\hline
\end{tabular}

of sample thickness variations, equation (1) is divided by the linear attenuation $\mu^{\star} d=\ln \left(I_{\mathrm{int}, 0} / I_{\mathrm{int}}\right)$ (where $I_{\mathrm{int}}$ and $I_{\text {int }, 0}$ are the transmitted intensities with and without sample). This yields a thickness-independent refraction value $C / \mu$ (called relative specific surface). The linear attenuation was obtained from X-ray transmission radiographs taken with a nominal pixel size of $3.6 \mu \mathrm{m}$ by $3.6 \mu \mathrm{m}$. The transmission radiographs were also used to calculated 2-D porosity maps of the samples. Further details on the experimental procedure of SXRR can be found in Laquai et al., ${ }^{26}$ Nellesen et al., ${ }^{29}$ and Cabeza. ${ }^{30}$

Because the analyzer crystal only distinguishes refracted X-rays within its scattering plane, the SXRR results depend on the orientation of the inner surfaces (defects, pores, etc.) with respect to the scattering vector of the analyzer crystal. Therefore, the samples were imaged in two orientations: once with the build direction lying within, i.e., parallel to, the scattering plane (henceforth called parallel orientation; see fig. 2 , left), and once rotated $90^{\circ}$ around the beam axis with the build direction perpendicular to the scattering plane (henceforth called perpendicular orientation; see fig. 2 , right).

\section{SXCT}

Furthermore, two of the samples (namely, A1 and A10) were subjected to an additional SXCT investigation also performed at BAMline with the aim to gain 3-D information on the very same defects observed by SXRR. For this purpose, a coupon with dimensions of $10 \mathrm{~mm}$ by $0.3 \mathrm{~mm}$ by $1.5 \mathrm{~mm}$ was cut from the plates. A template matching algorithm was used to identify the location of the radiographic projections from SXCT in the SXRR radiographs and thus ensure analysis of identical defects. Tomography data were acquired at X-ray energy of $40 \mathrm{keV}$ and with a nominal pixel size of $0.435 \mu \mathrm{m}$ by $0.435 \mu \mathrm{m}$. The field of view was $1.6 \mathrm{~mm}$ by $1.1 \mathrm{~mm}$.

FIG. 2 Schematic sketch of the sample orientation with respect to the scattering plane of the analyzer crystal.

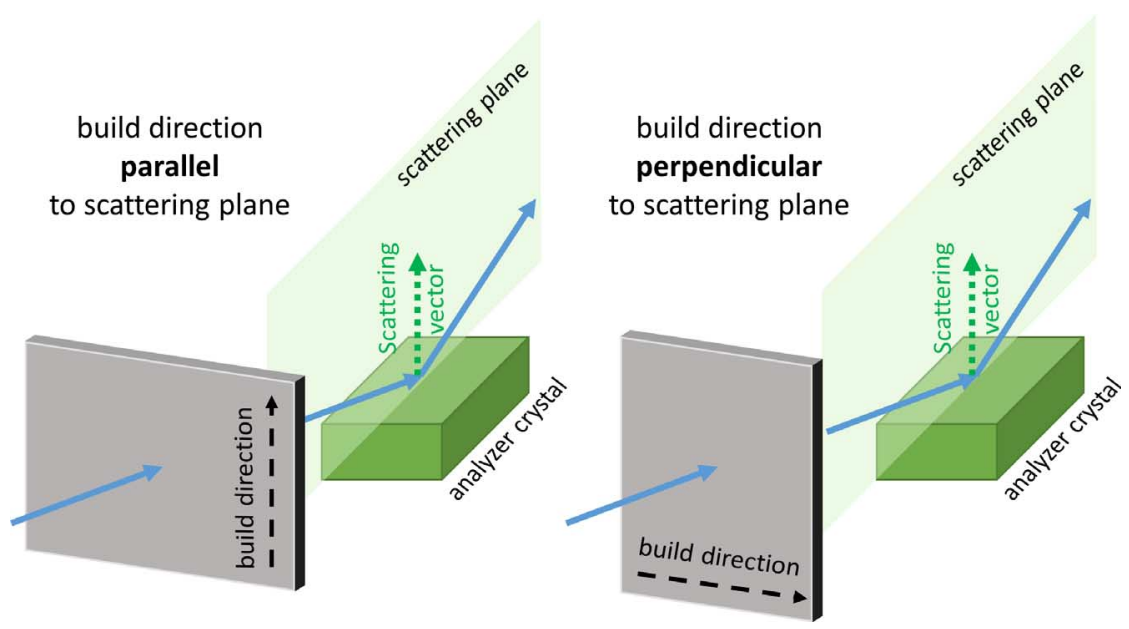




\section{Results and Discussion}

\section{TWO- AND THREE-DIMENSIONAL DEFECT IMAGING}

For the sake of clarity, only SXRR radiographs of samples A1 and A10 in both orientations are shown in figure 3. In sample A1 (fig. $3 A$ and $3 B ; E_{\mathrm{v}}=292 \mathrm{~J} \cdot \mathrm{mm}^{-3}$ ), a large number of empty pores can be recognized, as would be expected for high-energy density. We distinguish between empty pores and pores filled with unmolten powder. The defects are arranged parallel to the individual layers and distributed rather homogeneously over the sample. In Laquai et al., ${ }^{26}$ it was already reported that this kind of defect should appear as crescent shapes in the SXRR radiographs, because only the edges of the pores give rise to a refraction signal (indeed, the crescents are stacked in one direction, i.e., that of the scattering vector). These crescents are more obvious in the enlarged details presented in figure $4 A$ and $4 B$. The region shown in figure 4 is identical to the region investigated with SXCT, and a 3-D rendering of the marked region is shown as well in figure $4 C$. The direct comparison of SXRR and SXCT of the same defects proves that the crescents indeed originate from empty pores. Furthermore, there is no qualitative difference between the SXRR radiographs in perpendicular and parallel orientation; roughly the same amount of defects can be recognized in the two images (fig. $4 A$ and $4 B$ ), and the refraction values do not significantly differ, as would be expected for pores of roughly spherical shape. The ratio $C / \mu$ between perpendicular and parallel sample orientation amounts to 0.6 for most defects, i.e., the pores are not ideally spherical; in particular, large pores appear slightly elongated in the plane of the deposited layers. This deviation from a spherical shape can also be recognized from a 3-D sphericity analysis of the SXCT reconstructions. ${ }^{19,31}$ The sphericity (defined as $\Psi=\frac{6 \cdot \pi^{\frac{1}{2}} \cdot V}{A^{\frac{3}{2}}}$, where $V=$ volume and $A=$ area) ranges from 0.45 to 0.65 for the majority of the pores.

FIG. 3 SXRR radiographs of A1 and A10 samples (production parameters are also indicated); ( $A$ and $C$ ) scattering plane perpendicular to build direction (i.e., defect surfaces oriented parallel to the build direction are detected); $(B$ and $D$ ) scattering plane parallel to build direction (i.e., defect surfaces oriented perpendicular to the build direction are detected).

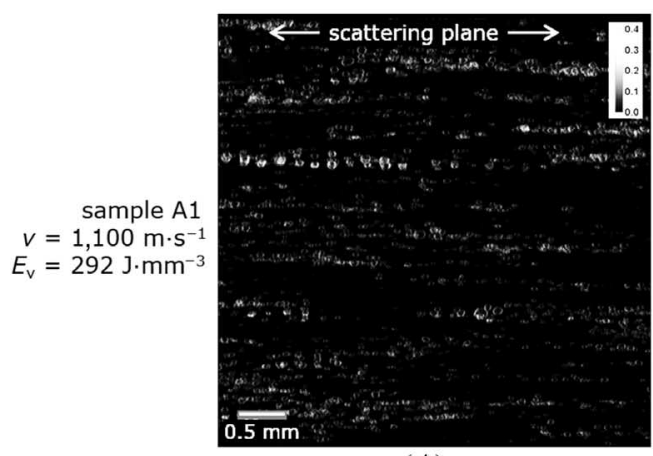

(A)

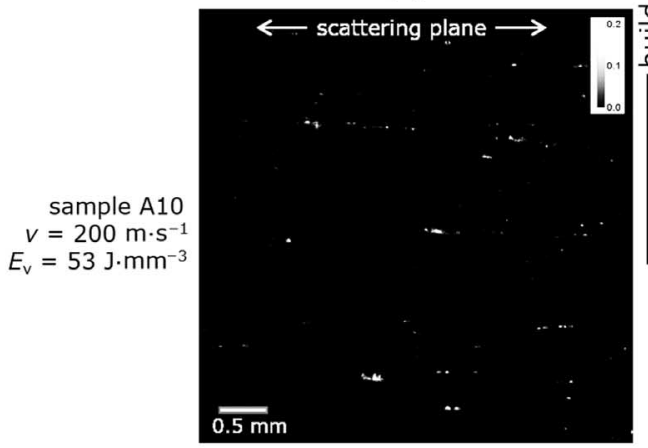

(C)

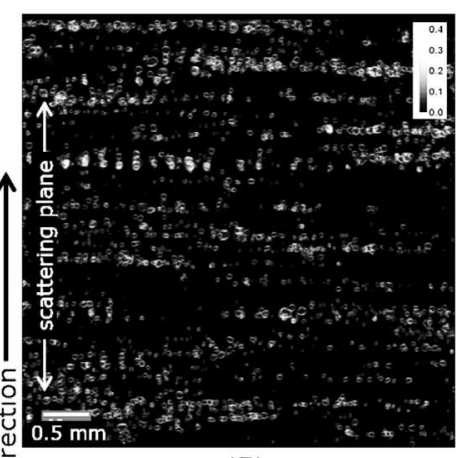

(B)

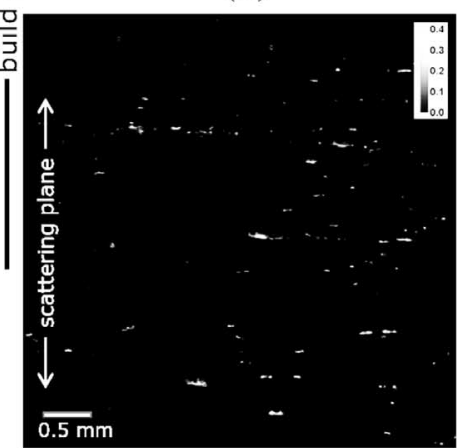

(D) 
FIG. 4

Enlarged detail of SXRR radiographs of sample $A 1$ $\left(E_{\mathrm{v}}=292 \mathrm{~J} \cdot \mathrm{mm}^{-3}\right)$ in (A) perpendicular orientation and

(B) parallel orientation with (C) 3-D rendering of highlighted defects from SXCT.

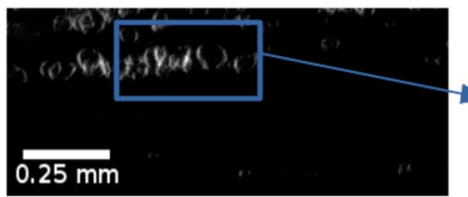

(A)

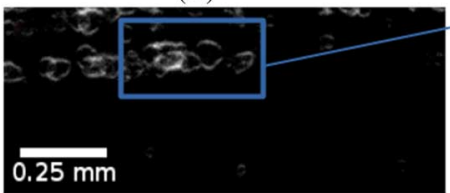

(B)

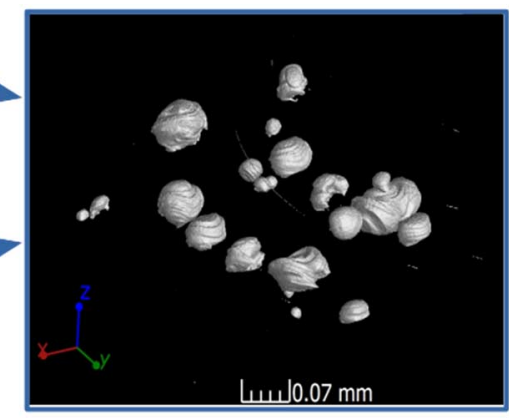

(C)

Sample A10 $\left(E_{\mathrm{v}}=53 \mathrm{~J} \cdot \mathrm{mm}^{-3}\right.$; fig. $3 C$ and $\left.3 D\right)$ shows a different type of defects. The bright spots indicate voids with an internal structure and complex shape. In Laquai et al., ${ }^{26}$ these were already attributed to fusing defects and balling. This is now further corroborated by a direct comparison between SXRR and SXCT of the same defects (see fig. 5). The SXCT data clearly reveal the voids created by balling, and even unmolten powder particles can be identified in large voids. The complex shapes lead to smaller 3-D sphericities of these defects: the sphericity ranges between 0.2 and 0.3 . Furthermore, sample A10 shows a significant difference of $C / \mu$ between perpendicular and parallel scattering orientation. Even on a qualitative level, it is obvious that many more defects are detected, and their refraction value is generally higher in the parallel orientation (note the different gray levels in fig. 3C and 3D). This clearly indicates that the defects are not only arranged parallel to the layers of production

FIG. 5 Enlarged detail of SXRR radiographs of sample A10 (53 J.mm $\mathrm{mm}^{-3}$ ) in $(A)$ perpendicular orientation and $(B)$ parallel orientation with 3-D rendering of highlighted defects from SXCT (I-III indicate the same defect type in SXRR and SXCT).

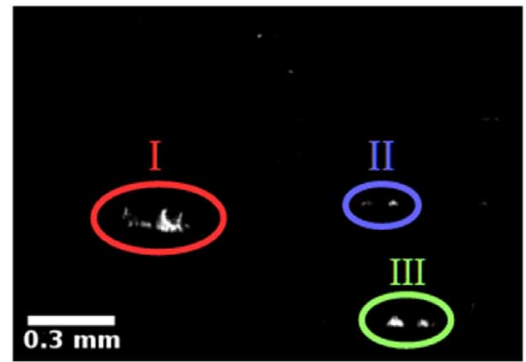

(A)

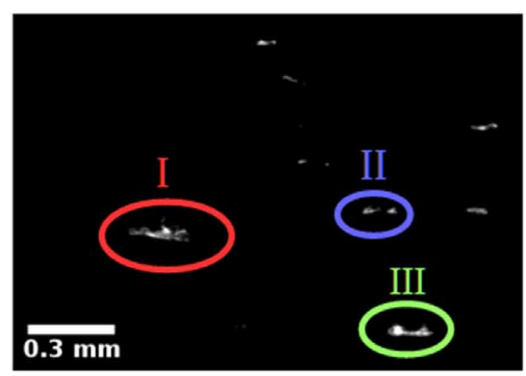

(B)
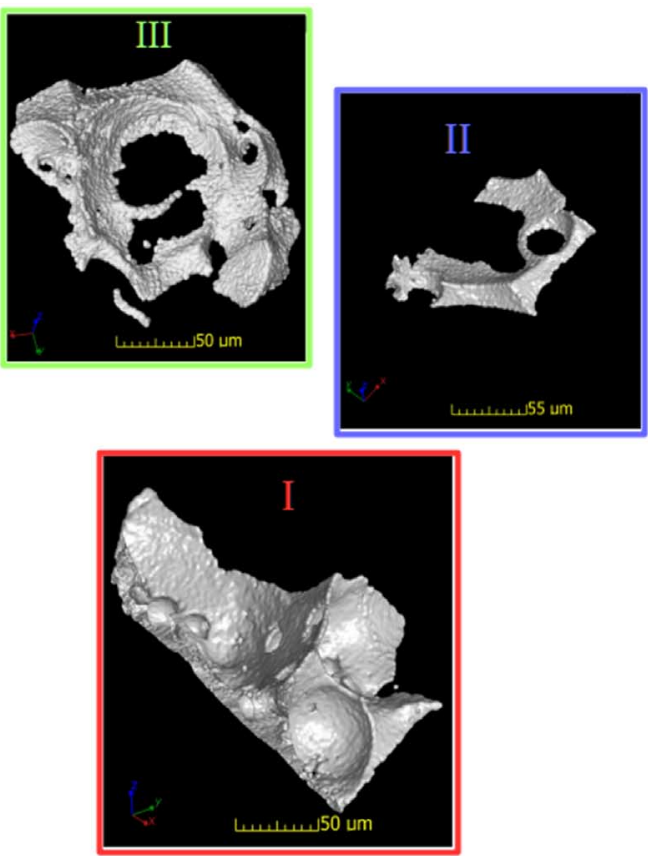
but also that the surfaces of the defects are oriented in this plane. A quantitative evaluation shows that the ratio between perpendicular and parallel orientation amounts to 0.02 for most defects.

\section{QUANTITATIVE POROSITY AND RELATIVE SPECIFIC SURFACE DETERMINATION}

It is interesting (and a good-practice "sanity check") to compare the determination of porosity by means of different methods. Figure 6 presents the results of total porosity from Archimedes' weighing, optical microscopy, $\mathrm{X}$-ray computed tomography (CT), and X-ray radiography. For all methods, except Archimedes' weighing, a minimum of porosity can clearly be observed around an energy density of $120 \mathrm{~J} \cdot \mathrm{mm}^{-3}$ (sample A4). The values from absorption-based X-ray radiography yield the smallest porosity values but deviate only marginally from the results of optical microscopy. SXCT yields generally higher porosity values than the 2-D methods, but significant deviations can only be recognized for low-energy densities. We must bear in mind, when comparing the porosity values from the different methods, that the captured measurement volumes are quite different. For example, SXCT can have a 10 times better spatial resolution than SXRR, but SXRR can investigate up to 20 times larger volume. If we look at figure $3 C$ and $3 D$, it is possible that SXCT was randomly selected for either a high or lowdefect density volume.

Indeed, SXRR porosity detection can be considered as the most precise of the methods used. The main error sources affecting the porosity value for each detector pixel are (a) the (theoretical) density of the fully dense material and (b) the number of gray values in the image (typically 256). The total error is therefore similar to that of the Archimedes' and SXCT methods and lies around 1\%. However, when calculating the average over the whole image (comparable with a macroscopic measurement), a large number (typically in excess of $10^{6}$ ) of pixels is taken, and therefore, the total error becomes much smaller.

Figure 7 shows the mean value of the relative specific surface $C / \mu$ of the segmented defects. At low-energy densities, the defects show a higher value of $C / \mu$ : fusing defects, which are predominant for low $E_{\mathrm{v}}$, exhibit a larger amount of free surfaces because of their complex shape and to powder particles trapped within the voids. Moreover, there is a marked difference between the parallel and perpendicular orientation. In parallel orientation, $C / \mu$ is larger than in perpendicular orientation, meaning that the surfaces are mostly aligned with the layers of the production process. Increasing the energy density leads to a decrease of $C / \mu$, and the difference between the two orientations becomes smaller. This means that at higher energy densities, the proportion of spherical keyhole pores increases. At high-energy densities, in which the amount of keyhole pores is higher than that of fusing defects, $C / \mu$ is roughly the same for both orientations, as would be expected for spherical voids. The increase of $C / \mu$ in sample A1 $\left(292 \mathrm{~J} / \mathrm{mm}^{-3}\right)$ with respect to A2 $\left(195 \mathrm{~J} / \mathrm{mm}^{-3}\right)$ can be explained by its high defect density. As can be recognized in the radiographs (fig. $3 A$ and $3 B$ ), the individual defects at different depths overlap in the

FIG. 6

Total porosity calculated from absorption-based radiographs, $\mathrm{X}$-ray $\mathrm{CT}$, optical microscopy, and Archimedes' weighing.

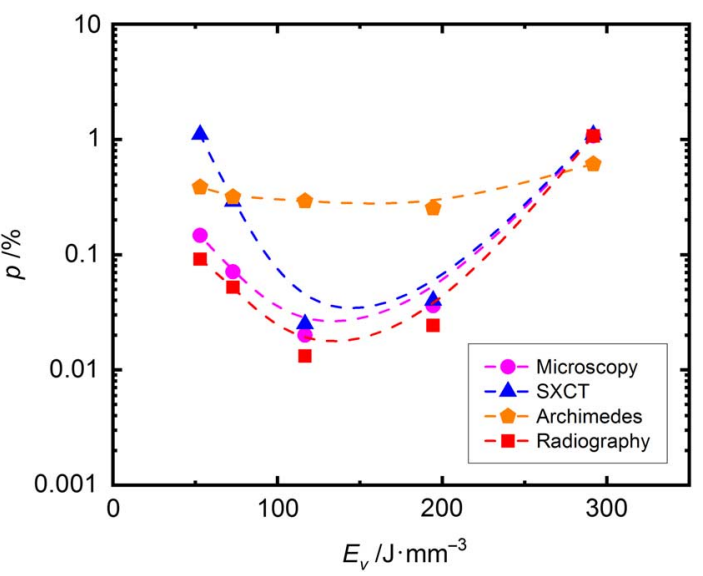


FIG. 7

Mean value of $\mathrm{C} / \mu$ of segmented defects calculated from SXRR.

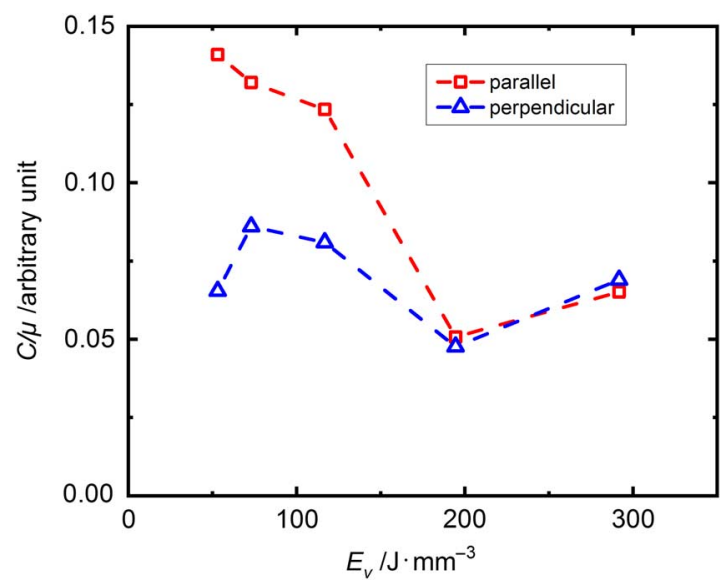

$\mathrm{X}$-ray projection and cannot be distinguished from each other. Furthermore, it can be recognized that the minimum of $C / \mu$ and the minimum of total porosity do not coincide. At $E_{\mathrm{v}} \approx 120 \mathrm{~J} \cdot \mathrm{mm}^{-3}$ (the minimum of porosity), $\mathrm{C} / \mu$, especially in parallel orientation, is not at its minimum. This fact and the large difference between parallel and perpendicular orientation indicate that fusing defects are the predominant defect type, which has been shown to be more detrimental to the mechanical properties. ${ }^{3,32}$

\section{Classification of Defects}

The results shown indicate that the refraction value can be used successfully to distinguish keyhole pores from binding defects, even when the individual shape cannot be discerned because of the limited image resolution. While the aforementioned analysis gives an evaluation of global values, in order to gain insight into the distribution of the different defect types, a classification of the individual defects would be necessary.

To quantify the difference between different kinds of pores, the ratio between $C / \mu$ in two perpendicular orientations was chosen.

$$
q=\frac{\left(\frac{C}{\mu}\right)_{\text {perpendicular }}}{\left(\frac{\mathrm{C}}{\mu}\right)_{\text {parallel }}}
$$

In order to classify these different types of pores, a threshold of $C / \mu$ between different pore types is needed. A reliable threshold can only be determined using a statistically significant number of objects (pores). Determining this threshold for each image (sample) is impractical, since the porosity of the samples produced in the optimum energy density range is too small to offer reliable statistics. Moreover, if our classification tool aims at being applicable to any sample, we ought to determine a global threshold (for the material under investigation). Therefore, the threshold value was determined from the analysis of samples A1 and A10 and then used for all other samples (we will show A2 as an example). In fact, $\mathrm{A} 1$ and $\mathrm{A} 10$ were produced with very low and very high $E_{\mathrm{v}}$, respectively, so they mainly contain crack-like and keyhole pores, respectively. The ratio was calculated for each segmented defect in the two samples, and frequency histograms are shown in figure 8. Since there is a significant number of defects that are detected in the parallel orientation but not in the perpendicular orientation, choosing $C / \mu$ in parallel orientation as the denominator avoided undefined data points. The position of the maxima of each distribution was determined by fitting a Pearson VII distribution to the respective histogram. The maxima were found to be $q=0.58$ for sample A1 and $q=0.02$ for sample A10. A reasonable threshold value to distinguish the two types of pores was set halfway between the two maxima, at $q=0.3$. 
FIG. 8

Frequency histogram of the ratio $q=(C / \mu)$ perpendicular $/(C / \mu)$ parallel of segmented defects.

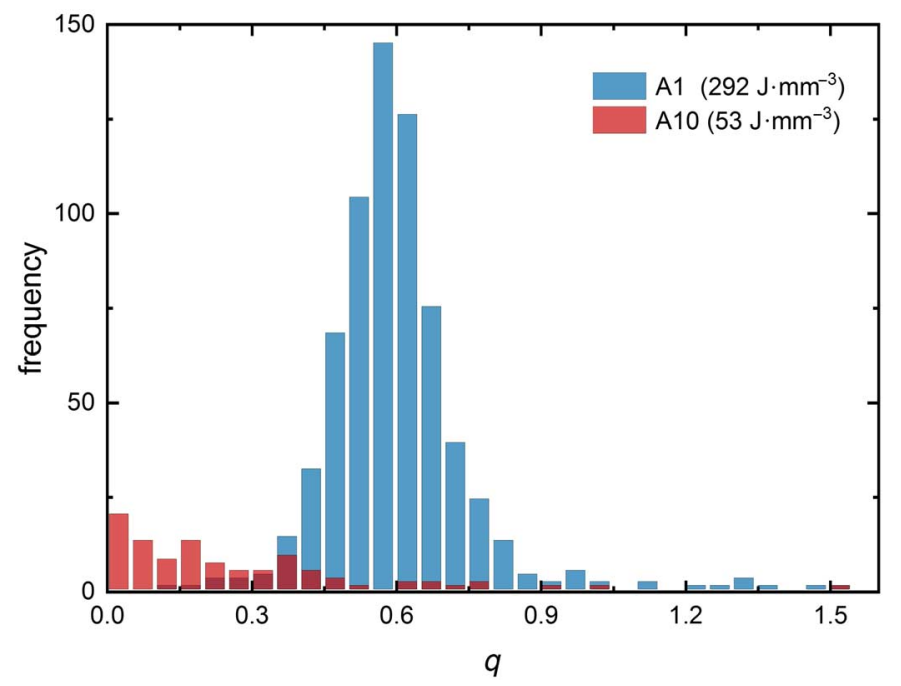

FIG. 9

Sum of SXRR

radiographs of the two orientations of sample A2 $\left(E \vee=117 \mathrm{~J} \cdot \mathrm{mm}^{-3}\right)$ overlaid with the results of the classification (see legend).

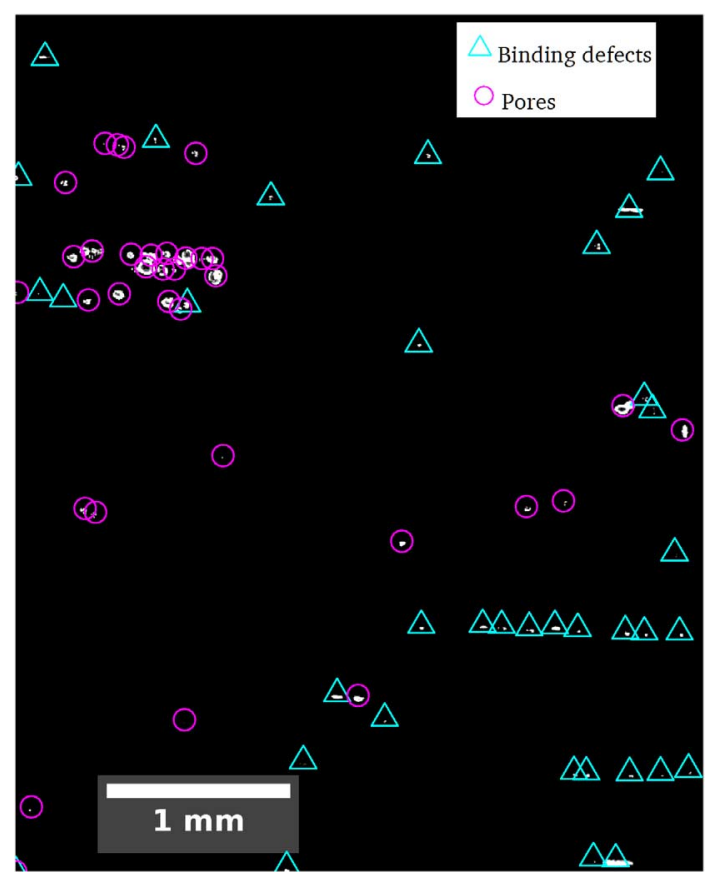

This threshold value was used to classify the defects in a third sample (namely, A2) into "pores" and "binding defects." The result of the classification procedure is shown in figure 9. The image shows the result of adding the SXRR radiographs obtained in the two orientations (this simplifies the visual recognition of the defect shape) and overlaying markers representing the result of the automated classification. The classification is in good agreement with visual inspection (manual classification). From those defects for which a visual classification from the shape is possible, only four pores were falsely classified as binding defects by our algorithm, and there were no 
binding defects falsely classified as pores. That represented a total of $6 \%$ false classifications. We reached therefore a confidence level of $94 \%$ for correct defect classification.

In conclusion, we introduced a valuable tool to aid characterization and quality assessment of AM materials (and parts) through defect classification. The classification is not based on measures that rely on image resolution and is therefore applicable to larger samples (or parts). This tool is a prelude to standardization of AM defects, analogous to what has been already defined in the field of cast metallic parts and welds. ${ }^{33}$ Besides its scientific interest in the field of materials performance (fatigue and mechanical properties in general depend on defect shape), the tool has therefore an enormous industrial value.

\section{Conclusions}

A combination of SXRR and SXCT allowed for characterizing and classifying the porosity in Ti-6Al-4V produced by SLM. We show by a direct comparison between SXRR and SXCT that the detectability of defects in SXRR is at least as good as that of SXCT (which possesses a 10 times better spatial resolution, however), while the volume

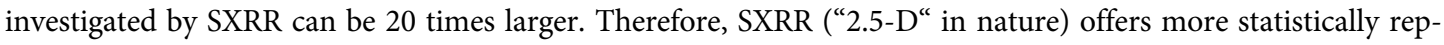
resentative results with less experimental effort and well complements 3-D imaging techniques.

Since keyhole pores and fusing defects cause different X-ray refraction signals, we could distinguish different types of voids. The ratio of the so-called relative internal specific surface $(C / \mu)$ in two orientations $\left(90^{\circ}\right.$ rotation) can serve as a simple shape descriptor, which was successfully used here to classify defects in a reliable manner. In this way, it is possible to either assess the quality of a part using global values or gain information about the spatial distribution of the different defects within the sample.

\section{ACKNOWLEDGMENTS}

This work was financially supported by Bundesanstalt für Materialforschung und -prüfung (BAM) within the Menschen-Ideen-Strukturen program [Ideen_2013_25]. The Deutsches Zentrum für Luft-und Raumfahrt acknowledges the basic funding received from the German Ministry of Economy and Energy (Bundesministerium für Wirtschaft und Energie). We thank Helmholtz-Zentrum Berlin colleagues for their support as well as Ralf Britzke and Thomas Wolk (BAM) for their assistance during beam time at BAMline. DLR thanks Ahmet Turak and Tarik Merzouk for the manufacturing of the SLM samples.

\section{References}

1. C. Y. Yap, C. K. Chua, Z. L. Dong, Z. H. Liu, D. Q. Zhang, L. E. Loh, and S. L. Sing, "Review of Selective Laser Melting: Materials and Applications," Applied Physics Reviews 2, no. 4 (December 2015): 041101. https://doi.org/10.1063/1. 4935926

2. L. Thijs, F. Verhaeghe, T. Craeghs, J. V. Humbeeck, and J.-P. Kruth, “A Study of the Microstructural Evolution during Selective Laser Melting of Ti-6Al-4V,” Acta Materialia 58, no. 9 (May 2010): 3303-3312. https://doi.org/10.1016/j. actamat.2010.02.004

3. S. Leuders, S. Meiners, L. Wu, A. Taube, T. Tröster, and T. Niendorf, "Structural Components Manufactured by Selective Laser Melting and Investment Casting-Impact of the Process Route on the Damage Mechanism under Cyclic Loading," Journal of Materials Processing Technology 248 (October 2017): 130-142. https://doi.org/10.1016/j.jmatprotec.2017. 04.026

4. T. Vilaro, C. Colin, and J. D. Bartout, "As-Fabricated and Heat-Treated Microstructures of the Ti-6Al-4V Alloy Processed by Selective Laser Melting," Metallurgical and Materials Transactions A 42, no. 10 (October 2011): 3190-3199. https://doi. org/10.1007/s11661-011-0731-y

5. H. Gong, K. Rafi, H. Gu, G. D. J. Ram, T. Starr, and B. Stucker, "Influence of Defects on Mechanical Properties of Ti-6Al4V Components Produced by Selective Laser Melting and Electron Beam Melting," Materials \& Design 86 (December 2015): 545-554. https://doi.org/10.1016/j.matdes.2015.07.147

6. J. Günther, D. Krewerth, T. Lippmann, S. Leuders, T. Tröster, A. Weidner, H. Biermann, and T. Niendorf, "Fatigue Life of Additively Manufactured Ti-6Al-4V in the Very High Cycle Fatigue Regime," International Journal of Fatigue 94 (January 2017): 236-245. https://doi.org/10.1016/j.ijfatigue.2016.05.018

7. S. Leuders, M. Thöne, A. Riemer, T. Niendorf, T. Tröster, H. A. Richard, and H. J. Maier, "On the Mechanical Behaviour of Titanium Alloy TiAl6V4 Manufactured by Selective Laser Melting: Fatigue Resistance and Crack Growth Performance," International Journal of Fatigue 48 (March 2013): 300-307. https://doi.org/10.1016/j.ijfatigue.2012.11.011 
8. S. A. Khairallah, A. T. Anderson, A. Rubenchik, and W. E. King, "Laser Powder-Bed Fusion Additive Manufacturing: Physics of Complex Melt Flow and Formation Mechanisms of Pores, Spatter, and Denudation Zones," Acta Materialia 108 (April 2016): 36-45. https://doi.org/10.1016/j.actamat.2016.02.014

9. S. Leuders, T. Lieneke, S. Lammers, T. Tröster, and T. Niendorf, "On the Fatigue Properties of Metals Manufactured by Selective Laser Melting - The Role of Ductility,” Journal of Materials Research 29, no. 17 (September 2014): 1911-1919. https://doi.org/10.1557/jmr.2014.157

10. S. Leuders, M. Vollmer, F. Brenne, T. Tröster, and T. Niendorf, "Fatigue Strength Prediction for Titanium Alloy TiAl6V4 Manufactured by Selective Laser Melting," Metallurgical and Materials Transactions A 46, no. 9 (September 2015): 38163823. https://doi.org/10.1007/s11661-015-2864-x

11. R. M. Kabir and H. Richter, "Modeling of Processing-Induced Pore Morphology in an Additively-Manufactured Ti-6Al4V Alloy," Materials 10, no. 2 (February 2017): 145. https://doi.org/10.3390/ma10020145

12. B. Vrancken, L. Thijs, J.-P. Kruth, and J. V. Humbeeck, "Heat Treatment of Ti6Al4V Produced by Selective Laser Melting: Microstructure and Mechanical Properties," Journal of Alloys and Compounds 541 (November 2012): 177-185. https:// doi.org/10.1016/j.jallcom.2012.07.022

13. V. Cain, L. Thijs, J. V. Humbeeck, B. V. Hooreweder, and R. Knutsen, "Crack Propagation and Fracture Toughness of Ti6Al4V Alloy Produced by Selective Laser Melting,” Additive Manufacturing 5 (January 2015): 68-76. https://doi.org/10. 1016/j.addma.2014.12.006

14. G. Bruno, A. M. Efremov, A. N. Levandovskyi, and B. Clausen, "Connecting the Macro- and Microstrain Responses in Technical Porous Ceramics: Modeling and Experimental Validations," Journal of Materials Science 46, no. 1 (January 2011): 161-173. https://doi.org/10.1007/s10853-010-4899-0

15. G. Bruno and M. Kachanov, "Porous Microcracked Ceramics under Compression: Micromechanical Model of Non-linear Behavior," Journal of the European Ceramics Society 33, no. 11 (October 2013): 2073-2085. https://doi.org/10.1016/j. jeurceramsoc.2013.03.012

16. W. Xu, S. Sun, J. Elambasseril, Q. Liu, M. Brandt, and M. Qian, “Ti-6Al-4V Additively Manufactured by Selective Laser Melting with Superior Mechanical Properties," JOM 67, no. 3 (March 2015): 668-673. https://doi.org/10.1007/s11837015-1297-8

17. V. Chastand, A. Tezenas, Y. Cadoret, P. Quaegebeur, W. Maia, and E. Charkaluk, "Fatigue Characterization of Titanium Ti-6Al-4V Samples Produced by Additive Manufacturing," Procedia Structural Integrity 2 (2016): 3168-3176. https://doi. org/10.1016/j.prostr.2016.06.395

18. G. Kasperovich and J. Hausmann, "Improvement of Fatigue Resistance and Ductility of TiAl6V4 Processed by Selective Laser Melting," Journal of Materials Processing Technology 220 (June 2015): 202-214. https://doi.org/10.1016/j. jmatprotec.2015.01.025

19. G. Kasperovich, J. Haubrich, J. Gussone, and G. Requena, "Correlation between Porosity and Processing Parameters in TiAl6V4 Produced by Selective Laser Melting," Materials \& Design 105 (September 2016): 160-170. https://doi.org/10. 1016/j.matdes.2016.05.070

20. M. P. Hentschel, R. Hosemann, A. Lange, B. Uther, and R. Brückner, "Small-Angle X-ray Refraction on Metal Wires, Glass Filaments and Hard Elastic Polypropylene” (in German), Acta Crystallographica A 43, no. 4 (1987): 506-513. https://doi.org/10.1107/S0108767387099100

21. B. R. Müller, A. Lange, M. Harwardt, and M. P. Hentschel, "Synchrotron-Based Micro-CT and Refraction-Enhanced Micro-CT for Non-destructive Materials Characterisation," Advanced Engineering Materials 11, no. 6 (June 2009): 435-440. https://doi.org/10.1002/adem.200800346

22. B. R. Müller and M. P. Hentschel, "Micro-Diagnostics: X-ray and Synchrotron Techniques," in Handbook of Technical Diagnostics: Fundamentals and Application to Structures and Systems, ed. H. Czichos (Berlin: Springer, 2013), 287-300.

23. B. R. Müller, R. C. Cooper, A. Lange, A. Kupsch, M. Wheeler, M. P. Hentschel, A. Staude, A. Pandey, A. Shyam, and G. Bruno, "Stress-Induced Microcrack Density Evolution in $\beta$-Eucryptite Ceramics: Experimental Observations and Possible Route to Strain Hardening," Acta Materialia 144 (February 2018): 627-641. https://doi.org/10.1016/j.actamat.2017.10.030

24. A. Kupsch, B. R. Müller, A. Lange, and G. Bruno, "Microstructure Characterisation of Ceramics via 2D and 3D X-ray Refraction Techniques,” Journal of the European Ceramic Society 37, no. 5 (May 2017): 1879-1889. https://doi.org/10. 1016/j.jeurceramsoc.2016.12.031

25. A. Kupsch, A. Lange, M. P. Hentschel, Y. Onel, T. Wolk, A. Staude, K. Ehrig, B. R. Müller, and G. Bruno, "Evaluating Porosity in Cordierite Diesel Particulate Filter Materials, Part 1 X-ray Refraction," Journal of Ceramic Science and Technology 4, no. 3 (2013): 169-175.

26. R. Laquai, B. R. Müller, G. Kasperovich, J. Haubrich, G. Requena, and G. Bruno, "X-ray Refraction Distinguishes Unprocessed Powder from Empty Pores in Selective Laser Melting Ti-6Al-4V," Materials Research Letters 6, no. 2 (2018): 130-135. https://doi.org/10.1080/21663831.2017.1409288

27. W. Görner, M. P. Hentschel, B. R. Müller, H. Riesemeier, M. Krumrey, G. Ulm, W. Diete, U. Klein, and R. Frahm, "BAMline: The First Hard X-ray Beamline at BESSY II," Nuclear Instruments and Methods in Physics Research Section A: Accelerators, Spectrometers, Detectors and Associated Equipment 467-468 (July 2001): 703-706. https://doi. org/10.1016/S0168-9002(01)00466-1

28. A. Rack, S. Zabler, B. R. Müller, H. Riesemeier, G. Weidemann, A. Lange, J. Goebbels, M. Hentschel, and W. Goerner, "High Resolution Synchrotron-Based Radiography and Tomography Using Hard X-rays at the BAMline (BESSY II)," 
Nuclear Instruments and Methods in Physics Research Section A: Accelerators, Spectrometers, Detectors and Associated Equipment 586, no. 2 (February 2008): 327-344. https://doi.org/10.1016/j.nima.2007.11.020

29. J. Nellesen, R. Laquai, B. R. Müller, A. Kupsch, M. P. Hentschel, N. B. Anar, E. Soppa, W. Tillmann, and G. Bruno, "In Situ Analysis of Damage Evolution in an $\mathrm{Al} / \mathrm{Al}_{2} \mathrm{O}_{3} \mathrm{MMC}$ under Tensile Load by Synchrotron X-ray Refraction Imaging," Journal of Materials Science 53, no. 8 (2018): 6021-6032. https://doi.org/10.1007/s10853-017-1957-x

30. S. Cabeza, B. R. Müller, R. Pereyra, R. Fernández, G. González-Doncel, and G. Bruno, "Evidence of Damage Evolution during Creep of Al-Mg Alloy Using Synchrotron X-ray Refraction,” Journal of Applied Crystallography 51, no. 2 (April 2018): 420-427. https://doi.org/10.1107/S1600576718001449

31. T. Thiede, T. Mishurova, S. Evsevleev, I. Serrano-Munoz, C. Gollwitzer, and G. Bruno, "3D Shape Analysis of Powder for Laser Beam Melting by Synchrotron X-ray CT,” Quantum Beam Science 3, no. 1 (February 2019): 3. https://doi.org/10. 3390/qubs3010003

32. P. Li, D. H. Warner, A. Fatemi, and N. Phan, "Critical Assessment of the Fatigue Performance of Additively Manufactured Ti-6Al-4V and Perspective for Future Research,” International Journal of Fatigue 85 (April 2016): 130-143. https://doi. org/10.1016/j.ijfatigue.2015.12.003

33. Welding and Allied Processes-Classification of Geometric Imperfections in Metallic Materials-Part 1: Fusion Welding, DIN EN ISO 6520-1:2007-11 (Berlin: Beuth Verlag, 2011). 